\title{
Topological properties for a perturbed first order sweeping process
}

\author{
Doria Affane \\ LMPA Laboratory, \\ Department of Mathematics, \\ Jijel University, Algeria \\ email: affanedoria@yahoo.fr
}

\author{
Loubna Boulkemh \\ LMPA Laboratory, \\ Department of Mathematics, \\ Jijel University, Algeria \\ email: 1.boulkemh@gmail.com
}

\begin{abstract}
In this paper, we consider a perturbed sweeping process for a class of subsmooth moving sets. The perturbation is general and takes the form of a sum of a single-valued mapping and a set-valued mapping. In the first result, we study some topological proprieties of the attainable set, the set-valued mapping considered here is upper semi-continuous with convex values. In the second result, we treat the autonomous problem under assumptions that do not require the convexity of the values and that weaken the assumption on the upper semi-continuity. Then, we deduce a solution of the time optimality problem.
\end{abstract}

\section{Introduction}

The attainable sets plays an important role in control theory; many problems of optimization, dynamics, planning procedures in mathematical economy and game theory can be stated and solved in terms of attainable sets. The perturbed state-dependent sweeping process is an evolution differential inclusion governed by the normal cone to a mobile set depending on both time and state variables, of the following form:

$$
\left\{\begin{array}{c}
-\dot{u}(t) \in N_{C(t, u(t))}(u(t))+F(t, u(t)), \text { a.e } t \in\left[T_{0}, T\right] \\
x(t) \in C(t, u(t)), \forall t \in\left[T_{0}, T\right] ; u\left(T_{0}\right)=u_{0} \in C\left(T_{0}, u_{0}\right)
\end{array}\right.
$$

2010 Mathematics Subject Classification: 34A60, 28A25

Key words and phrases: sweeping process, subsmooth sets, perturbation, almost convex, attainable sets, time optimal control problems 
where $N_{C(t, u(t))}(u(t))$ is the normal cone to $C(t, u(t))$ at $u(t)$ and $F$ is a setvalued or single-valued mapping playing the role of a perturbation to the problem, that is an external force applied on the system. This type of problems was initiated by J. J. Moreau in the 1970's and extensively studied by himself when the sets $\mathrm{C}(\mathrm{t})$ are assumed to be convex and $\mathrm{F} \equiv\{0\}$ (see $[24,25,26,27])$. The original motivation is to model quasistatic evolution in elastoplasticity, friction dynamics, granular material, contact dynamics. However, many applications of the sweeping processes can be also found nowadays in nonsmooth mechanics, convex optimization, modeling of crowd motion, mathematical economics, dynamic networks, switched electrical circuits, etc, see for example $[2,15,16,19,22]$ and the references therein. Existence (and possibly uniqueness) of solutions of such systems and their classical variants subjected to perturbation forces, state-dependent, second order sweeping processes, etc, have been studied fruitfully in the literature see for example $[1,3,7,8,9,10,11,14,17,21,22,28,29,30,31]$ and the references therein.

In [12], a generalization of convexity has been defined, that is the almost convexity of sets, the authors have shown the existence of solution to the upper semi-continuous differential inclusions $\dot{x}(t) \in F(x(t)), x(0)=a$. This almost convexity condition has been used successfully by $[3,4,5]$ to study the perturbed first order Moreau's sweeping process, the right-hand side contains a set-valued perturbation with almost convex values.

In this work, we extend the results in [3] in many direction. At first, we study in finite dimensional space, the existence of solution and the compactness of the attainable sets for the problem

$$
\left\{\begin{array}{l}
\dot{u}(t) \in-N_{C(t, u(t))}(u(t))+F(t, u(t))+f(t, u(t)), \quad \text { a.e. } t \in\left[T_{0}, T\right] \\
u(t) \in C(t, u(t)), \quad \forall t \in\left[T_{0}, T\right] ; \quad u\left(T_{0}\right)=u_{0} \in C\left(T_{0}, u_{0}\right),
\end{array}\right.
$$

when $F$ is a set-valued mapping with nonempty closed convex values, upper semi-continuous and the element of minimum norm satisfies a linear growth condition, $f$ is a continuous single-valued mapping and the moving sets $C(t, x)$ are equi-uniformlt-subsmooth. It is important to emphasize that this class of sets, introduced by D. Aussel, A. Daniilidis and L. Thibault in [6], is an extension of convexity and prox-regularity of a set. In this way, the result concerning existence of solution of the first order differential inclusion is more general. Second, we define a larger class contains set-valued mappings with almost convex values and their translated, then we study the existence of 
solution to the autonomous problem

$$
(\mathcal{A S P}) \quad\left\{\begin{array}{l}
\dot{u}(\mathrm{t}) \in-\mathrm{N}_{\mathrm{C}(\mathrm{u}(\mathrm{t}))}(\mathrm{u}(\mathrm{t}))+\mathrm{F}(\mathrm{u}(\mathrm{t}))+\mathrm{f}(\mathrm{u}(\mathrm{t})), \text { a.e. } \mathrm{t} \in\left[\mathrm{T}_{0}, \mathrm{~T}\right] ; \\
\mathrm{u}(\mathrm{t}) \in \mathrm{C}(\mathrm{u}(\mathrm{t})), \quad \forall \mathrm{t} \in\left[\mathrm{T}_{0}, \mathrm{~T}\right] ; \quad \mathrm{u}\left(\mathrm{T}_{0}\right)=\mathrm{u}_{0} \in \mathrm{C}\left(\mathrm{u}_{0}\right),
\end{array}\right.
$$

under the weaker assumption on the upper semi-continuity and the almost convexity of the values of $F$. We mention that $C, F$ and $f$ are assumed time independent for purely technical reasons. As will be shown, our almost convexity does not imply that the set of solutions to $(\mathcal{A S P})$ is compact in the space of continuous functions with uniform convergence, as happens in the case of the assumption of convexity, but only that the sections of this set of solutions are compact. As an application, we consider the autonomous control system

$$
\left(\mathcal{A S P}_{\mathcal{O}}\right)\left\{\begin{array}{l}
\dot{\mathrm{u}}(\mathrm{t}) \in-\mathrm{N}_{\mathrm{C}(\mathrm{u}(\mathrm{t}))}(\mathrm{u}(\mathrm{t}))+\mathrm{h}(\mathrm{u}(\mathrm{t}), z(\mathrm{t}))+\mathrm{f}(\mathrm{u}(\mathrm{t})), \text { a.e. } \mathrm{t} \in\left[\mathrm{T}_{0}, \mathrm{~T}\right] \\
z(\mathrm{t}) \in \mathrm{U}(\mathrm{u}(\mathrm{t})), \mathrm{u}(\mathrm{t}) \in \mathrm{C}(\mathrm{u}(\mathrm{t})), \forall \mathrm{t} \in\left[\mathrm{T}_{0}, \mathrm{~T}\right] ; \mathrm{u}\left(\mathrm{T}_{0}\right)=\mathrm{u}_{0} \in \mathrm{C}\left(\mathrm{u}_{0}\right),
\end{array}\right.
$$

controlled by parameters $z(t) \in \mathrm{U}(\mathrm{u}(\mathrm{t}))$, where $\mathrm{U}: \mathbb{R}^{n} \rightrightarrows \mathbb{R}^{n}$ is a set-valued mapping with compact values that is upper semi-continuous on $\mathbb{R}^{n}$. Under the almost convexity assumption on the sets

$$
F(u(t))=h(u(t), u(u(t)))=\{h(u(t), z(t))\}_{z(t) \in u(u(t))}
$$

and $\mathrm{F}(\mathrm{u}(\mathrm{t}))+\mathrm{f}(\mathrm{u}(\mathrm{t}))$ the solutions of the control problem $\left(\mathcal{A S \mathcal { P } _ { \mathcal { O } }}\right)$ are solutions to the $(\mathcal{A S P})$, in which the controls do not appear explicitly, we say that $\mathrm{F}$ is parameterized by elements of $\mathrm{U}$. The equivalence between a control system and the corresponding differential inclusion is the central idea used to prove the existence of solution to the minimum time problem for $\left(\mathcal{A S \mathcal { P } _ { \mathcal { O } }}\right)$. This paper is organized as follows: in the first section, we introduce preliminaries and background. In the second, we study the existence of solution to the problem $(\mathcal{S P})$ and some topological proprieties of the attainable set when the perturbation is convex. In the last section, we prove the existence of solution for a differential inclusion $(\mathcal{A S P})$ with almost convex perturbation and we deduce a solution of the time optimality problem.

\section{Preliminaries and background}

Throughout this paper $\mathbf{R}^{\mathfrak{n}}$ is the $\mathfrak{n}$-dimensional Euclidean space, $\mathcal{I}=\left[\mathrm{T}_{0}, \mathrm{~T}\right]$ $\left(T>T_{0} \geq 0\right)$ an interval of $\mathbf{R}, \mathbf{B}$ is the closed unit ball centered at the origin of $\mathbf{R}^{n}$ and $\mathbf{B}(a, \eta)$ the open ball of center $a$ and radius $\eta>0$. We denote 
by $\mathcal{C}_{\mathbf{R}^{n}}(\mathcal{I})$ the Banach space of all continuous maps from $\mathcal{I}$ into $\mathbf{R}^{\mathrm{n}}$ endowed with the sup-norm, $L_{\mathbf{R}^{n}}^{1}(\mathcal{I})$ stands for the space of all Lebesgue integrable $\mathbf{R}^{n}$ valued mappings defined on $\mathcal{I}$. A map $u: \mathcal{I} \rightarrow \mathbf{R}^{\mathfrak{n}}$ is absolutely continuous if there is a mapping $g \in \mathrm{L}_{\mathbf{R}^{n}}^{1}(\mathcal{I})$ such that $u(t)=u\left(T_{0}\right)+\int_{T_{0}}^{t} g(s) d s$, for all $t \in \mathcal{I}$. For a nonempty closed subset $K$ of $\mathbf{R}^{\mathrm{n}}, \operatorname{co}(\mathrm{K})$ (resp. $\overline{\mathbf{c o}}(\mathrm{K})$ ) stands for the convex (resp. closed convex) hull of $K$, which can be characterized by $\overline{\mathrm{co}}(\mathrm{K})=\left\{x \in \mathbf{R}^{\mathfrak{n}}, \forall x^{\prime} \in \mathbf{R}^{\mathfrak{n}},\left\langle x^{\prime}, x\right\rangle \leq \delta^{*}\left(x^{\prime}, K\right)\right\}$ where $\delta^{*}\left(x^{\prime}, K\right)=\sup _{y \in K}\left\langle x^{\prime}, y\right\rangle$ is the support function of $K$ at $x^{\prime} \in \mathbf{R}^{n}$. We denote by $d_{K}(\cdot)$ the usual distance function associated with $K$, i.e., $d_{K}(x)=\inf _{y \in K}\|x-y\|, \operatorname{Proj}_{K}(x)=\{y \in K$ : $\left.d_{K}(x)=\|x-y\|\right\}$ the projection set of $x$ into $K$ and by $m(K)=\operatorname{Proj}_{K}(0)$ the element of $\mathrm{K}$ with minimal norm, it is unique whenever $\mathrm{K}$ is a closed convex. If $\mathrm{F}$ is a measurable set-valued mapping, with nonempty closed convex values, then $F$ admits a measurable selection with minimal norm $m(F(x))=\operatorname{Proj}_{F(x)}(0)$. We will need the concept of Clarke subdifferential and normal cone. For a locally Lipschitzian function $\varphi: \mathbf{R}^{\mathfrak{n}} \rightarrow \mathbf{R} \cup\{\infty\}$, the Clarke subdifferential $\partial \varphi(\chi)$ of $\varphi$ at $\chi$ is the nonempty convex compact subset of $\mathbf{R}^{\mathfrak{n}}$, given by $($ see[13])

$$
\partial \varphi(x)=\left\{\xi \in \mathbf{R}^{n}:\langle\xi, v\rangle \leq \varphi^{o}(x, v), \text { for all } v \in \mathbf{R}^{\mathfrak{n}}\right\},
$$

where $\varphi^{\mathrm{o}}(\mathrm{x}, v)=\limsup _{\substack{\mathrm{y} \rightarrow \mathrm{x} \\ \mathrm{t} \downarrow \mathrm{O}}} \frac{\varphi(\mathrm{y}+\mathrm{t} v)-\varphi(\mathrm{y})}{\mathrm{t}}$ is the generalized directional derivative of $\varphi$ at $x$ in the direction $v$. The Clarke normal cone $N_{K}(x)$ at $x \in K$ is defined from $\mathrm{T}_{\mathrm{K}}^{\mathrm{C}}$ by polarity, that is,

$$
\mathrm{N}_{\mathrm{K}}(x)=\left\{\xi \in \mathbf{R}^{\mathrm{n}}:\langle\xi, v\rangle \leq 0, \text { for all } v \in \mathrm{T}_{\mathrm{K}}^{\mathrm{C}}(x)\right\},
$$

where $\mathrm{T}_{\mathrm{K}}^{\mathrm{C}}(x)$ is the Clarke tangent cone at $x \in \mathrm{K}$ given by $\mathrm{T}_{\mathrm{K}}^{\mathrm{C}}(x)=\left\{v \in \mathbf{R}^{\mathrm{n}}\right.$ : $\left.\mathrm{d}_{\mathrm{K}}^{\mathrm{o}}(\mathrm{x}, v)=0\right\}$.

The concept of Fréchet subdifferential will be needed. A vector $v \in \mathbf{R}^{\mathrm{n}}$ is a Fréchet subdifferential $\partial^{\mathrm{F}} \varphi(x)$ of $\varphi$ at $\chi$ (see[23]) provided that for every $\varepsilon>0$, there exists $\delta>0$ such that

$$
\langle\nu, y-x\rangle \leq \varphi(y)-\varphi(x)+\varepsilon\|y-x\|, \text { for all } y \in \mathbf{B}(x, \delta) .
$$

We always have the inclusion $\partial^{F} \varphi(x) \subset \partial \varphi(x)$, for all $x \in K$. The Fréchet normal cone at $x \in K$ is given by $N_{K}^{F}(x)=\partial^{F} \psi_{K}(x)$, where $\psi_{K}$ is the indicator function of $K$, that is, $\psi_{K}(x)=0$ if $x \in K$ and $\psi_{K}(x)=+\infty$ otherwise. So we have the inclusion $\mathrm{N}_{\mathrm{K}}^{\mathrm{F}}(\mathrm{x}) \subset \mathrm{N}_{\mathrm{K}}(\mathrm{x})$, for all $\mathrm{x} \in \mathrm{K}$. On the other hand, the 
Fréchet normal cone is also related (see[23]) to the Fréchet subdifferential of the distance function, since for all $x \in K$

$$
\partial^{\mathrm{F}} \mathrm{d}_{\mathrm{K}}(x)=\mathrm{N}_{\mathrm{K}}^{\mathrm{F}}(x) \cap \mathbf{B} .
$$

An important property is that, whenever $y \in \operatorname{Proj}_{K}(x)$, one has

$$
x-y \in N_{K}^{F}(y) \quad \text { hence also } x-y \in N_{K}(y) .
$$

Now, we introduce a class of subsmooth sets introduced in [6].

Definition 1 Let $\mathrm{K}$ be a closed subset of $\mathbf{R}^{\mathrm{n}}$. The set $\mathrm{K}$ is called subsmooth at $\mathrm{x}_{0} \in \mathrm{K}$, if for every $\varepsilon>0$ there exists $\delta>0$, such that for all $\mathrm{x}_{1}, \mathrm{x}_{2} \in$ $\mathbf{B}\left(x_{0}, \delta\right) \cap \mathrm{K}$ and $\xi_{i} \in \mathrm{N}_{\mathrm{K}}\left(\mathrm{x}_{\mathrm{i}}\right) \cap \mathbf{B}(\mathrm{i} \in\{1,2\})$, on has

$$
\left\langle\xi_{1}-\xi_{2}, x_{1}-x_{2}\right\rangle \geq-\varepsilon\left\|x_{1}-x_{2}\right\| .
$$

The set $\mathrm{K}$ is subsmooth, if it subsmooth at each point of $\mathrm{K}$. We say that $\mathrm{K}$ is uniformly subsmooth, if for every $\varepsilon>0$ there exists $\delta>0$, such that (3) holds for all $\mathrm{x}_{1}, \mathrm{x}_{2} \in \mathrm{K}$ satisfying $\left\|\mathrm{x}_{1}-\mathrm{x}_{2}\right\|<\delta$ and all $\xi_{i} \in \mathrm{N}_{\mathrm{K}}\left(\mathrm{x}_{\mathrm{i}}\right) \cap \mathbf{B}(\mathrm{i} \in\{1,2\})$.

The following subdifferential regularity of the distance function remains true for subsmooth sets (see [6]).

Proposition 1 Let $\mathrm{K}$ be a closed set of $\mathbf{R}^{\mathrm{n}}$. If $\mathrm{K}$ is subsmooth at $\mathrm{x} \in \mathrm{K}$, then

$$
\mathrm{N}_{\mathrm{K}}(\mathrm{x})=\mathrm{N}_{\mathrm{K}}^{\mathrm{F}}(\mathrm{x}) \quad \text { and } \quad \partial \mathrm{d}_{\mathrm{K}}(\mathrm{x})=\partial^{\mathrm{F}} \mathrm{d}_{\mathrm{K}}(\mathrm{x}) .
$$

The concept of equi-uniformly subsmoothness will also be helpful.

Definition 2 Let $(\mathrm{K}(\mathbf{q}))_{\mathrm{q} \in \mathrm{Q}}$ be a family of closed sets of $\mathbf{R}^{\mathrm{n}}$ with parameter $\mathrm{q} \in \mathrm{Q}$. This family is called equi-uniformly subsmooth, if for every $\varepsilon>0$, there exists $\delta>0$ such that, for each $\mathrm{q} \in \mathrm{Q}$, the inequality (3) holds for all $\mathrm{x}_{1}, \mathrm{x}_{2} \in \mathrm{K}(\mathrm{q})$ satisfying $\left\|\mathrm{x}_{1}-\mathrm{x}_{2}\right\|<\delta$ and all $\xi_{\mathrm{i}} \in \mathrm{N}_{\mathrm{K}(\mathrm{q})}\left(\mathrm{x}_{\mathrm{i}}\right) \cap \mathbf{B}$.

The next proposition provides partial upper semi-continuity property. For the proof, we refer the reader to [21].

Proposition 2 Let $\left\{\mathrm{K}(\mathrm{t}, \mathrm{x}):(\mathrm{t}, \mathrm{x}) \in \mathcal{I} \times \mathbf{R}^{\mathfrak{n}}\right\}$ be a family of nonempty closed sets of $\mathbf{R}^{n}$, which is equi-uniformly-subsmooth and let a real $\eta \geq 0$. Assume that there exist a real constants $\mathrm{L}_{1} \geq 0, \mathrm{~L}_{2} \in\left[0,1\right.$ [ such that, for any $\mathrm{x}_{1}, \mathrm{x}_{2}, \mathrm{y} \in$ $\mathbf{R}^{\mathrm{n}}$ and $\mathrm{t}, \mathrm{s} \in \mathcal{I}$

$$
\left|d_{K\left(t, x_{1}\right)}(y)-d_{K\left(s, x_{2}\right)}(y)\right| \leq L_{1}|t-s|+L_{2}\left\|x_{1}-x_{2}\right\| .
$$

Then, the following assertions hold: 
(i) for all $(\mathrm{t}, \mathrm{x}, \mathrm{y}) \in \mathrm{Gph} \mathrm{K}$, we have $\eta \partial \mathrm{d}_{\mathrm{K}(\mathrm{t}, \mathrm{x})}(\mathrm{y}) \subset \eta \mathbf{B}$;

(ii) for any sequence $\left(t_{\mathfrak{n}}, x_{\mathfrak{n}}\right)_{\mathfrak{n}}$ in $\mathcal{I} \times \mathbf{R}^{\mathfrak{n}}$ converging to $(\mathrm{t}, \mathrm{x})$, any $\left(\mathrm{y}_{\mathrm{n}}\right)_{\mathrm{n}}$ converging to $\mathrm{y} \in \mathrm{K}(\mathrm{t}, \mathrm{x})$ with $\mathrm{y}_{\mathrm{n}} \in \mathrm{K}\left(\mathrm{t}_{\mathrm{n}}, \mathrm{x}_{\mathrm{n}}\right)$ and any $\xi \in \mathbf{R}^{\mathrm{n}}$, we have

$$
\limsup _{n \rightarrow+\infty} \delta^{*}\left(\xi, \eta \partial d_{K\left(t_{n}, x_{n}\right)}\left(y_{n}\right)\right) \leq \delta^{*}\left(\xi, \eta \partial d_{K(t, x)}(y)\right) .
$$

In the next, we give the definition of the almost convex sets and attainable sets.

Definition 3 [12] For a vector space $\mathrm{X}$, a set $\mathrm{D} \subset \mathrm{X}$ is called almost convex if for every $\xi \in \operatorname{co}(\mathrm{D})$ there exist $\lambda_{1}$ and $\lambda_{2}, 0 \leq \lambda_{1} \leq 1 \leq \lambda_{2}$ such that $\lambda_{1} \xi \in \mathrm{D}$ and $\lambda_{2} \xi \in \mathrm{D}$.

Any convex set is almost convex since $D=c o(D)$. If $Q$ is a convex set not containing the origin, $\mathrm{D}=\partial \mathrm{Q}$ is almost convex, and if the convex set $\mathrm{Q}$ contains the origin, one take $\mathrm{D}=\{0\} \cup \partial \mathrm{Q}$. The origin plays a particular role in the definition of almost convexity. It ensues that the class of almost convex sets is not stable under translation, for example the set for example the set $\mathrm{K}=\{0,1\}$ is almost convex, while $\mathrm{K}-\frac{1}{2}=\left\{-\frac{1}{2}, \frac{1}{2}\right\}$ is not.

Definition 4 The attainable set of any problem at time $\tau \in \mathcal{I}$ is defined by

$$
\mathcal{R}_{\mathrm{u}_{0}}(\tau)=\left\{\chi \in \mathbf{R}^{\mathfrak{n}}: \boldsymbol{x}=\mathfrak{u}(\tau) \text { such that } \mathfrak{u}(\cdot) \in \mathcal{S}_{\tau}\left(\mathbf{u}_{0}\right)\right\},
$$

where $\mathcal{S}_{\tau}\left(\mathrm{u}_{0}\right)$ is the set of the trajectories of our problem on the interval $\left[\mathrm{T}_{0}, \mathrm{t}\right]$.

We will also need the following result, which is a discrete version of Gronwall's Lemma.

Lemma 1 Let $\alpha>0,\left(a_{n}\right)$ and $\left(b_{n}\right)$ two nonnegative sequence such that

$$
a_{n} \leq \alpha+\sum_{k=0}^{n-1} b_{k} a_{k}, \quad \text { for all } n \in N
$$

Then, for every $\mathrm{n} \in \mathbf{N}^{*}$, we have

$$
a_{n} \leq \alpha \exp \left(\sum_{k=0}^{n-1} b_{k}\right)
$$




\section{Convex case}

In this section, we study the existence of solution and some topological properties of the attainable set for the sweeping process $(\mathcal{S P})$ when $\mathrm{F}$ is an upper semi-continuous set-valued mapping with nonempty closed convex values unnecessarily bounded.

Theorem 1 Let $\mathrm{C}: \mathcal{I} \times \mathbf{R}^{\mathrm{n}} \rightarrow \mathbf{R}^{\mathrm{n}}$ be a set-valued mapping with nonempty closed values satisfying:

$\left(\mathcal{A}_{1}^{\mathrm{C}}\right)$ for all $(\mathrm{t}, \mathrm{x}) \in \mathcal{I} \times \mathbf{R}^{\mathrm{n}}$, the sets $\mathrm{C}(\mathrm{t}, \mathrm{x})$ are equi-uniformly subsmooth;

$\left(\mathcal{A}_{2}^{\mathrm{C}}\right)$ there are two constants $\mathrm{L}_{1} \geq 0, \mathrm{~L}_{2} \in[0,1$ [ such that, for all $\mathrm{t}, \mathrm{s} \in \mathcal{I}$ and any $\mathrm{x}, \mathrm{u}, \boldsymbol{v} \in \mathbf{R}^{\mathrm{n}}$ on has

$$
\left|d_{C(t, u)}(x)-d_{C(s, v)}(x)\right| \leq L_{1}|t-s|+L_{2}|| u-v \| .
$$

Let $\mathrm{F}: \mathcal{I} \times \mathbf{R}^{\mathrm{n}} \rightarrow \mathbf{R}^{\mathrm{n}}$ be a set-valued mapping with nonempty closed convex values, upper semi-continuous such that:

$\left(\mathcal{A}^{\mathrm{F}}\right)$ for some real $\alpha \geq 0, \mathrm{~d}_{\mathrm{F}(\mathrm{t}, \mathrm{x})}(0) \leq \alpha(1+\|\mathrm{x}\|)$, for all $(\mathrm{t}, \mathrm{x}) \in \mathcal{I} \times \mathbf{R}^{\mathrm{n}}$. And $\mathrm{f}: \mathcal{I} \times \mathbf{R}^{\mathrm{n}} \rightarrow \mathbf{R}^{\mathrm{n}}$ be a continuous mapping such that:

$\left(\mathcal{A}^{f}\right)$ for some real $\beta>0,\|\mathrm{f}(\mathrm{t}, \mathrm{x})\| \leq \beta(1+\|x\|)$, for all $(\mathrm{t}, \mathrm{x}) \in \mathcal{I} \times \mathbf{R}^{\mathrm{n}}$.

Then, for any $\mathrm{u}_{0} \in \mathrm{C}\left(0, \mathrm{u}_{0}\right)$

(1) the problem $(\mathcal{S P})$ admits a Lipschitz solution;

(2) for $\tau \in \mathcal{I}$ fixed, the attainable set $\mathcal{R}_{\mathrm{u}_{0}}(\tau)$ is compact;

(3) the set-valued mapping $\mathcal{R}_{\mathrm{u}_{0}}(\cdot)$ is upper semi-continuous.

Proof. (1) The existence of solution: for each $(t, x) \in \mathcal{I} \times \mathbf{R}^{\mathrm{n}}$, we put $\mathrm{m}(\mathrm{t}, x)=$ $\operatorname{Proj}_{F(t, x)}(0)$ the element of minimal norm of $F$ and $h(t, x)=m(t, x)+f(t, x)$. It follows that, $\|h(t, x)\| \leq \gamma(1+\|x\|)$ with $\gamma=\alpha+\beta$. For each $n \in \mathbf{N}^{*}$, we consider a partition of $\mathcal{I}$ by $\mathcal{I}_{i}^{n}=\left[\mathrm{t}_{i}^{n}, \mathrm{t}_{i+1}^{n}\left[, \mathrm{t}_{i}^{\mathrm{n}}=\mathrm{T}_{0}+i \mu_{n}, \mu_{n}=\frac{T-T_{0}}{n}, i \in\right.\right.$ $\{0,1, \cdots, \mathrm{n}-1\}$ and $\mathcal{I}_{0}^{\mathrm{n}}=\left\{\mathbf{t}_{0}^{\mathrm{n}}\right\}=\left\{\mathrm{T}_{0}\right\}$.

Step 1. We define inductively the sequence $\left(x_{i}^{n}\right)_{0 \leq i \leq n}$ in $\mathbf{R}^{\mathfrak{n}}$. Putting $x_{0}^{n}=$ $\mathrm{u}_{0} \in \mathrm{C}\left(\mathrm{t}_{0}^{\mathfrak{n}}, x_{0}^{\mathfrak{n}}\right)$ and for each $i \in\{1,2, \cdots, n-1\}$ the following inclusions is well defined

$$
x_{i+1}^{n} \in C\left(t_{i+1}^{n}, x_{i}^{n}\right) \text {, }
$$




$$
x_{i}^{n}+\mu_{n} h\left(t_{i}^{n}, x_{i}^{n}\right)-x_{i+1}^{n} \in N_{C\left(t_{i+1}^{n}, x_{i}^{n}\right)}\left(x_{i+1}^{n}\right) .
$$

Indeed, for $i=0$ and since $C\left(t_{1}^{n}, x_{0}^{n}\right)$ has closed values, we can take

$$
x_{1}^{n} \in \operatorname{Proj}_{C\left(t_{1}^{n}, x_{0}^{n}\right)}\left(x_{0}^{n}+\mu_{n} h\left(t_{0}^{n}, x_{0}^{n}\right)\right),
$$

clearly

$$
x_{1}^{n} \in C\left(t_{1}^{n}, x_{0}^{n}\right)
$$

Then, by (2), we obtain

$$
x_{0}^{\mathfrak{n}}+\mu_{n} h\left(t_{0}^{n}, x_{0}^{n}\right)-x_{1}^{n} \in N_{C\left(t_{1}^{n}, x_{0}^{n}\right)}\left(x_{1}^{\mathfrak{n}}\right) .
$$

Using $\left(\mathcal{A}_{2}^{\mathrm{C}}\right)$ and (8), we get

$$
\begin{aligned}
\left\|x_{1}^{n}-x_{0}^{n}\right\| & \leq d_{C\left(t_{1}^{n}, x_{0}^{n}\right)}\left(x_{0}^{n}+\mu_{n} h\left(t_{0}^{n}, x_{0}^{n}\right)\right)+\mu_{n}\left\|h\left(t_{0}^{n}, x_{0}^{n}\right)\right\| \\
& \leq\left|d_{C\left(t_{1}^{n}, x_{0}^{n}\right)}\left(x_{0}^{n}\right)-d_{C\left(t_{0}^{n}, x_{0}^{n}\right)}\left(x_{0}^{n}\right)\right|+2 \mu_{n}\left\|h\left(t_{0}^{n}, x_{0}^{n}\right)\right\| \\
& \leq L_{1} \mu_{n}+2 \gamma \mu_{n}\left(1+\left\|x_{0}^{n}\right\|\right) .
\end{aligned}
$$

Assume that, for $i \in\{0,1, \cdots, n-1\}$ the points $x_{1}^{n}, x_{2}^{n}, \cdots, x_{i}^{n}$ have been constructed satisfying (6) and (7). Since $C\left(t_{i+1}^{n}, x_{i}^{n}\right)$ is closed, we can take

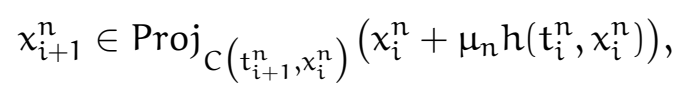

and

$$
x_{i+1}^{n} \in C\left(t_{i+1}^{n}, x_{i}^{n}\right) .
$$

Using the characterization of the normal cone in terms of projection operator, we can write a.e. $t \in \mathcal{I}$

$$
x_{i}^{n}+\mu_{n} h\left(t_{i}^{n}, x_{i}^{n}\right)-x_{i+1}^{n} \in N_{C\left(t_{i+1}^{n}, x_{i}^{n}\right)}\left(x_{i+1}^{n}\right) .
$$

By $\left(\mathcal{A}_{2}^{\mathrm{C}}\right)$ and (6), we get

$$
\begin{aligned}
\left\|x_{i+1}^{n}-x_{i}^{n}\right\| & \leq d_{C\left(t_{i+1}^{n}, x_{i}^{n}\right)}\left(x_{i}^{n}+\mu_{n} h\left(t_{i}^{n}, x_{i}^{n}\right)\right)+\left\|\mu_{n} h\left(t_{i}^{n}, x_{i}^{n}\right)\right\| \\
& \leq\left|d_{C\left(t_{i+1}^{n}, x_{i}^{n}\right)}\left(x_{i}^{n}\right)-d_{C\left(t_{i}^{n}, x_{i-1}^{n}\right)}\left(x_{i}^{n}\right)\right|+2 \mu_{n}\left\|h\left(t_{i}^{n}, x_{i}^{n}\right)\right\| \\
& \leq L_{1} \mu_{n}+L_{2}\left\|x_{i}^{n}-x_{i-1}^{n}\right\|+2 \gamma \mu_{n}\left(1+\left\|x_{i}^{n}\right\|\right) .
\end{aligned}
$$


By induction, we find for $i \in\{0,1, \cdots, n-1\}$,

$$
\left\|x_{i+1}^{n}-x_{i}^{n}\right\| \leq\left(L_{1}+2 \gamma\right) \mu_{n} \sum_{k=0}^{i} L_{2}^{k}+2 \gamma \mu_{n} \sum_{k=0}^{i} L_{2}^{i-k}\left\|x_{k}^{n}\right\|,
$$

since $\mathrm{L}_{2} \in[0,1[$, we get

$$
\left\|x_{i+1}^{n}-x_{i}^{n}\right\| \leq \frac{L_{1}+2 \gamma}{1-L_{2}} \mu_{n}+2 \gamma \mu_{n} \sum_{k=0}^{i} L_{2}^{i-k}\left\|x_{k}^{n}\right\| .
$$

Furthermore, we have

$$
\begin{aligned}
\left\|x_{i}^{n}-x_{0}^{n}\right\| & \leq\left\|x_{i}^{n}-x_{i-1}^{n}\right\|+\left\|x_{i-1}^{n}-x_{i-2}^{n}\right\|+\cdots+\left\|x_{1}^{n}-x_{0}^{n}\right\| \\
& \leq \frac{L_{1}+2 \gamma}{1-L_{2}} \mu_{n}+2 \gamma \mu_{n} \sum_{k=0}^{i-1} L_{2}^{i-k}\left\|x_{k}^{n}\right\|+\frac{L_{1}+2 \gamma}{1-L_{2}} \mu_{n} \\
& +2 \gamma \mu_{n} \sum_{k=0}^{i-2} L_{2}^{i-k}\left\|x_{k}^{n}\right\|+\cdots+\mu_{n}\left(L_{1}+2 \gamma\right)+2 \gamma \mu_{n}\left\|x_{0}^{n}\right\| \\
& \leq \frac{L_{1}+2 \gamma}{1-L_{2}} \mu_{n}(i-1)+2 \gamma \mu_{n}\left\|x_{0}^{n}\right\| \sum_{k=0}^{i-1} L_{2}^{k}+2 \gamma \mu_{n}\left\|x_{1}^{n}\right\| \sum_{k=0}^{i-1} L_{2}^{k} \\
& +2 \gamma \mu_{n}\left\|x_{2}^{n}\right\| \sum_{k=0}^{i-1} L_{2}^{k}+\cdots+2 \gamma \mu_{n}\left\|x_{i-1}^{n}\right\| \sum_{k=0}^{i-1} L_{2}^{k} \\
& \leq T \frac{L_{1}+2 \gamma}{1-L_{2}}+\frac{2 \gamma T}{1-L_{2}} \sum_{k=0}^{i-1}\left\|x_{k}^{n}\right\| .
\end{aligned}
$$

Then,

$$
\left\|x_{i}^{n}\right\| \leq\left\|x_{0}^{n}\right\|+T \frac{L_{1}+2 \gamma}{1-L_{2}}+\frac{2 \gamma T}{1-L_{2}} \sum_{k=0}^{i-1}\left\|x_{k}^{n}\right\| .
$$

By Lemma 1 and for all $i \in\{0,1, \cdots, n-1\}$, we can write

$$
\left\|x_{i}^{n}\right\| \leq\left(\left\|x_{0}^{n}\right\|+T \frac{L_{1}+2 \gamma}{1-L_{2}}\right) \exp \left(\frac{2 \gamma T}{1-L_{2}}\right)=\eta .
$$

Using relations (9) and (10), we get

$$
\left\|x_{i+1}^{n}-x_{i}^{n}\right\| \leq \frac{L_{1}+2 \gamma}{1-L_{2}} \mu_{n}+2 \gamma \mu_{n} \sum_{k=0}^{i} L_{2}^{i-k} \eta .
$$


Since $\mathrm{L}_{2} \in[0,1[$, we obtain

$$
\left\|x_{i+1}^{n}-x_{i}^{n}\right\| \leq \frac{1}{1-L_{2}} \mu_{n}\left(L_{1}+2 \gamma+2 \gamma \eta\right) .
$$

Step 2. Construction of sequence $\left(u_{n}(\cdot)\right)_{n>0}$.

For any $t \in \mathcal{I}_{i}^{n}$ with $i \in\{0,1, \cdots, n-1\}$ and for every $n \geq 1$, we define

$$
u_{n}(t)=x_{i}^{n}+\left(t-t_{i}^{n}\right) \frac{x_{i+1}^{n}-x_{i}^{n}}{\mu_{n}} .
$$

Observe that $u_{n}\left(t_{i}^{n}\right)=x_{i}^{n}$, and

$$
\dot{u}_{n}(t)=\frac{x_{i+1}^{n}-x_{i}^{n}}{\mu_{n}} .
$$

By (6) and (7) we can write

$$
\begin{gathered}
u_{n}\left(t_{i+1}^{n}\right) \in C\left(t_{i+1}^{n}, u_{n}\left(t_{i}^{n}\right)\right) \\
\dot{u}_{n}(t) \in-N_{C\left(t_{i+1}^{n}, u_{n}\left(t_{i}^{n}\right)\right)}\left(u_{n}\left(t_{i+1}^{n}\right)\right)+h\left(t_{i}^{n}, u_{n}\left(t_{i}^{n}\right)\right), \text { a.e. } t \in \mathcal{I}_{i}^{n} .
\end{gathered}
$$

Relations (11) and (13) imply that

$$
\left\|\dot{\mathrm{u}}_{n}(\mathrm{t})\right\| \leq \frac{1}{1-\mathrm{L}_{2}}\left(\mathrm{~L}_{1}+2 \gamma+2 \gamma \eta\right)=\Delta .
$$

Now let us defined the step functions from $\mathcal{I}$ to $\mathcal{I}$ by

$$
\begin{aligned}
& \theta_{n}(t)=\left\{\begin{array}{ccc}
t_{i}^{n} & \text { if } & t \in \mathcal{I}_{i}^{n}, \\
t_{n-1}^{n} & \text { if } & t=T_{0} .
\end{array}\right. \\
& \rho_{n}(t)=\left\{\begin{array}{clc}
t_{i+1}^{n} & \text { if } & t \in \mathcal{I}_{i}^{n}, \\
T & \text { if } & t=T .
\end{array}\right.
\end{aligned}
$$

Observe that, for all $t \in \mathcal{I}$,

$$
\lim _{n \rightarrow+\infty}\left|\theta_{n}(t)-t\right|=\lim _{n \rightarrow+\infty}\left|\rho_{n}(t)-t\right|=0 .
$$

Combining (14), (15), (17) and (18), it results

$$
\begin{gathered}
u_{n}\left(\rho_{n}(t)\right) \in C\left(\rho_{n}(t), u_{n}\left(\theta_{n}(t)\right)\right), \quad \text { for all } t \in \mathcal{I}, \\
\dot{u}_{n}(t) \in-N_{C}\left(\rho_{n}(t), u_{n}\left(\theta_{n}(t)\right)\right) \\
\left(u_{n}\left(\rho_{n}(t)\right)\right)+h\left(\theta_{n}(t), u_{n}\left(\theta_{n}(t)\right)\right), \text { a.e. } t \in \mathcal{I},
\end{gathered}
$$


Furthermore, for all $t \in \mathcal{I}$, we have

$$
\left\|h\left(\theta_{n}(t), u_{n}\left(\theta_{n}(t)\right)\right)\right\| \leq \gamma(1+\eta)=\Theta,
$$

and

$$
\left\|m\left(\theta_{n}(t), u_{n}\left(\theta_{n}(t)\right)\right)\right\| \leq \alpha(1+\eta)
$$

with $\mathfrak{m}\left(\theta_{\mathfrak{n}}(\cdot), u_{\mathfrak{n}}\left(\theta_{\mathfrak{n}}(\cdot)\right)\right)=\operatorname{Proj}_{\boldsymbol{F}\left(\theta_{\mathfrak{n}}(\cdot), u_{\mathfrak{n}}\left(\theta_{\mathfrak{n}}(\cdot)\right)\right.}(0)$.

Step 3. The convergence of the sequences.

By relation (12) and (16) we have for all $t \in \mathcal{I}$,

$\left\|u_{n}\left(\rho_{n}(t)\right)\right\|-\left\|u_{n}(t)\right\| \leq\left\|u_{n}\left(\rho_{n}(t)\right)-u_{n}(t)\right\| \leq\left\|\dot{u}_{n}(s)\right\|\left(\rho_{n}(t)-t\right) \leq \Delta\left(\rho_{n}(t)-t\right)$, then

$$
\lim _{n \rightarrow+\infty}\left\|u_{n}\left(\rho_{n}(t)\right)-u_{n}(t)\right\|=0
$$

In the same way

$$
\lim _{n \rightarrow+\infty}\left\|u_{n}\left(\theta_{n}(t)\right)-u_{n}(t)\right\|=0 .
$$

So, $\left(u_{n}(t)\right)_{n \geq 1}$ is relatively compact for all $t \in \mathcal{I}$, on the other hand $\left(u_{n}(\cdot)\right)_{n \geq 1}$ is equi-continuous according to (16). Using Ascoli-Arzelà's theorem, $\left(u_{n}(\cdot)\right)_{n \geq 1}$ is relatively compact in $\mathcal{C}_{\mathbf{R}^{\mathfrak{n}}}(\mathcal{I})$, so we can extract a subsequence of $\left(u_{\mathfrak{n}}(\cdot)\right)_{\mathfrak{n} \geq 1}$ (that we do not relabel) which converges uniformly to some mapping $\mathfrak{u}(\cdot) \in$ $\mathcal{C}_{\mathbf{R}^{\mathfrak{n}}}(\mathcal{I})$ and $\left(\dot{u}_{n}(\cdot)\right)_{n \geq 1}$ converges weakly in $\mathrm{L}_{\mathbf{R}^{\mathfrak{n}}}^{1}(\mathcal{I})$ to a mapping $y$ with $\|\mathrm{y}(\mathrm{t})\| \leq \Delta$. Fixing $\mathrm{t} \in \mathcal{I}$ and taking any $\xi \in \mathbf{R}^{\mathrm{n}}$, the above weak convergence in $\mathrm{L}_{\mathbf{R}^{\mathfrak{n}}}^{1}(\mathcal{I})$ yields

$$
\lim _{n \rightarrow+\infty} \int_{T_{0}}^{T}\left\langle x_{\mathcal{I}}(s) \xi, \dot{u}_{n}(s)\right\rangle d s=\int_{T_{0}}^{T}\left\langle x_{\mathcal{I}}(s) \xi, y(s)\right\rangle d s
$$

or equivalently

$$
\lim _{n \rightarrow+\infty}\left\langle\xi, u_{0}+\int_{T_{0}}^{t} \dot{u}_{n}(s) d s\right\rangle=\left\langle\xi, u_{0}+\int_{T_{0}}^{t} y(s) d s\right\rangle .
$$

Then, $\lim _{n \rightarrow+\infty} \int_{T_{0}}^{t} \dot{u}_{n}(s) d s=\int_{T_{0}}^{t} y(s)$ ds. Since $u_{n}(\cdot)$ is an of absolutely continuous mapping, we get

$$
\lim _{n \rightarrow+\infty}\left(u_{n}(t)-u_{0}\right)=\lim _{n \rightarrow+\infty} \int_{T_{0}}^{t} \dot{u}_{n}(s) d s=\int_{T_{0}}^{t} y(s) d s
$$

Then $u(t)=u_{0}+\int_{T_{0}}^{t} y(s) d s$ and $y=\dot{u}$.

Let set $\left(m\left(\theta_{n}(\cdot), u_{n}\left(\theta_{n}(\cdot)\right)\right)\right)_{n}=\left(p_{n}(\cdot)\right)_{n}$, for all $n \geq 0$, by (23) we get 
$\left\|p_{n}(t)\right\| \leq \alpha(1+\eta)$, which means that $\left(p_{n}\right)$ is integrably bounded, so, by extracting a subsequence, not relabeled, we may assume that $\left(p_{n}\right)$ weakly converges in $\mathrm{L}_{\mathbf{R}^{\mathfrak{n}}}^{1}(\mathcal{I})$ to some mapping $p \in \mathrm{L}_{\mathbf{R}^{\mathfrak{n}}}^{1}(\mathcal{I})$, with $\|p(t)\| \leq \alpha(1+\eta)$ for all $\mathrm{t} \in \mathcal{I}$.

Let put $\left(f\left(\theta_{\mathfrak{n}}(\cdot), u_{\mathfrak{n}}\left(\theta_{\mathfrak{n}}(\cdot)\right)\right)\right)_{\mathfrak{n}}=\left(\mathbf{q}_{\mathfrak{n}}(\cdot)\right)_{\mathfrak{n}}$, according to the continuity of $\mathrm{f}$, (19) and (25) we get that $\left(\mathbf{q}_{\mathfrak{n}}(\cdot)\right)$ converges to $\mathrm{q}(\cdot)$ and for all $\mathrm{t} \in \mathcal{I},\|\mathrm{q}(\mathrm{t})\| \leq$ $\beta(1+\eta)$.

Step 4 . We prove that the mapping $u$ is a solution of $(\mathcal{S P})$. Fix any $t \in \mathcal{I}$, by $\left(\mathcal{A}_{2}^{\mathrm{C}}\right)$ and $(20)$, we have

$$
\begin{aligned}
& d_{C(t, u(t))}\left(u_{n}(t)\right) \leq\left\|u_{n}(t)-u_{n}\left(\rho_{n}(t)\right)\right\|+d_{C(t, u(t))}\left(u_{n}\left(\rho_{n}(t)\right)\right) \\
& \leq\left\|u_{n}(t)-u_{n}\left(\rho_{n}(t)\right)\right\|+\left|d_{C(t, u(t))}\left(u_{n}\left(\rho_{n}(t)\right)\right)-d_{C\left(\rho_{n}(t), u_{n}\left(\theta_{n}(t)\right)\right)}\left(u_{n}\left(\rho_{n}(t)\right)\right)\right| \\
& \leq\left\|u_{n}\left(\rho_{n}(t)\right)-u_{n}(t)\right\|+L_{1}\left|t-\rho_{n}(t)\right|+L_{2}\left\|u(t)-u_{n}\left(\theta_{n}(t)\right)\right\| .
\end{aligned}
$$

Using (19), (24), (25), and by passing to the limit in the preceding inequality, thanks to the closedness of $\mathrm{C}(\mathrm{t}, \mathfrak{u}(\mathrm{t}))$, we get

$$
u(t) \in C(t, u(t)), \quad \text { for all } t \in \mathcal{I} .
$$

Furthermore, by (16) and (22), we have

$$
\left\|-\dot{u}_{n}(t)+p_{n}(t)+q_{n}(t)\right\| \leq \Delta+\Theta=\Upsilon .
$$

Then, (21) and (26) yield that

$$
-\dot{u}_{n}(t)+p_{n}(t)+q_{n}(t) \in N_{C\left(\rho_{n}(t), u_{n}\left(\theta_{n}(t)\right)\right.}\left(u_{n}\left(\rho_{n}(t)\right)\right) \cap \gamma_{B},
$$

from relation (1) and Proposition 1, we get

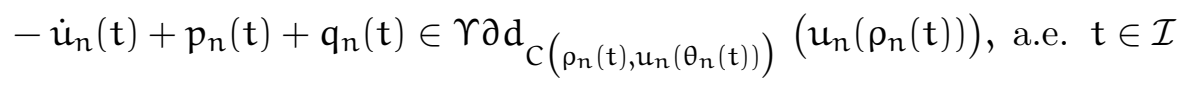

and

$$
p_{n}(t) \in F\left(\theta_{n}(t), u_{n}\left(\theta_{n}(t)\right)\right) .
$$

Since $\left(-\dot{u}_{n}+p_{n}+q_{n}, p_{n}\right)$ weakly converges in $L_{\mathbf{R}^{n} \times \mathbf{R}^{n}}^{1}(\mathcal{I})$ to $(-\dot{u}+p+q, p)$, by Mazur's Lemma, there exists a sequence $\left(\omega_{n}, \zeta_{n}\right)_{n \geq 1}$ with

$$
\omega_{n} \in \operatorname{co}\left\{-\dot{u}_{k}+p_{k}+q_{k}\right\} \quad \text { and } \quad \zeta_{n} \in \operatorname{co}\left\{p_{k}, k \geq n\right\}, n \geq 0
$$

such that $\left(\omega_{n}, \zeta_{n}\right)_{n \geq 1}$ converges strongly in $L_{\mathbf{R}^{n} \times \mathbf{R}^{n}}^{1}(\mathcal{I})$ to $(-\dot{u}+p+q, p)$. By extracting a subsequence if necessary, we suppose that $\left(\omega_{n}, \zeta_{n}\right)_{n \geq 1}$ converges 
a.e. to $(-\dot{u}+p+q, p)$. Then, there is a Lebesgue negligible set $\mathcal{S} \subset \mathcal{I}$ such that, for every $t \in \mathcal{I} \backslash \mathcal{S}$, on one hand $\left(\omega_{n}, \zeta_{n}\right)_{n \geq 1}$ converges strongly to $(-\dot{u}+p+q, p)$ and on the other hand the inclusions (27) and (28) hold true for every integer $n$ as well as the inclusions

$-\dot{u}(t)+p(t)+q(t) \in \bigcap_{n \geq 0} \overline{\left\{\omega_{k}(t), k \geq n\right\}} \subset \bigcap_{n \geq 0} \overline{c o}\left\{-\dot{u}_{k}(t)+p_{k}(t)+q_{k}(t), k \geq n\right\}$,

and

$$
p(t) \in \bigcap_{n \geq 0} \overline{\left\{p_{k}(t), \quad k \geq n\right\}} \subset \bigcap_{n \geq 0} \overline{c 0}\left\{p_{k}(t), \quad k \geq n\right\},
$$

Fix any $\mathrm{t} \in \mathcal{I} \backslash \mathcal{S}$ and $z \in \mathbf{R}^{\mathrm{n}}$ the relations (27) and (29) gives

$$
\langle z,-\dot{u}(t)+p(t)+q(t)\rangle \leq \limsup _{n \rightarrow+\infty} \delta^{*}\left(z, \gamma \partial d_{C\left(\rho_{n}(t), u_{n}\left(\theta_{n}(t)\right)\right)}\left(u_{n}\left(\rho_{n}(t)\right)\right)\right) .
$$

By Proposition 2, we get

$$
\langle z,-\dot{u}(t)+p(t)+q(t)\rangle \leq \delta^{*}\left(z, \gamma_{\partial} d_{C(t, u(t))}(u(t))\right) .
$$

Since $r \partial d_{c(t, u(t))}(u(t))$ is closed convex values, we obtain

$$
-\dot{u}(t)+p(t)+q(t) \in \operatorname{rod}_{C(t, u(t))}(u(t)) \subset N_{C(t, u(t))}(u(t)) .
$$

Furthermore, according to (28), (30) and the upper semi-continuous of F, we have

$$
\langle z, p(t)\rangle \leq \limsup _{n \rightarrow+\infty} \delta^{*}\left(z, F\left(\theta_{n}(t), u_{n}\left(\theta_{n}(t)\right)\right) \leq \delta^{*}(z, F(t, u(t))) .\right.
$$

Since $F$ has closed convex values, we conclude that $p(t) \in F(t, u(t))$ for all $\mathrm{t} \in \mathcal{I} \backslash \mathcal{S}$. By $(31)$

$$
\dot{u}(t) \in-N_{C(t, u(t))}(u(t))+F(t, u(t))+f(t, u(t)) \text {, a.e. } t \in \mathcal{I} \text {. }
$$

2) It suffice to show that the solution set

$$
S_{\tau}\left(u_{0}\right)=\left\{u \in \mathcal{C}_{\mathbf{R}^{n}}\left(\left[T_{0}, \tau\right]\right): u \text { is a Lipschitz solution of }(\mathcal{S P})\right\}
$$

is compact for $\tau \in \mathcal{I}$. By part 1 , we have $S_{\tau}\left(u_{0}\right) \neq \emptyset$. Let $\left(u_{n}\right)_{n}$ be a sequence in $S_{\tau}\left(u_{0}\right)$. Then, for each $n \in \mathbf{N}, u_{n}$ is a Lipschitz solution of $(\mathcal{S P})$ with

$$
\left\|\dot{\mathrm{u}}_{\mathrm{n}}(\tilde{\mathrm{t}})\right\| \leq \Delta \text {, a.e. } \tilde{\mathrm{t}} \in\left[\mathrm{T}_{0}, \tau\right],
$$


and

$$
\left\|\mathrm{u}_{n}(\tilde{\mathrm{t}})\right\| \leq\left\|\mathrm{u}_{0}\right\|+\int_{\mathrm{T}_{0}}^{\tilde{\mathrm{t}}}\left\|\dot{\mathrm{u}}_{\mathrm{n}}(\mathrm{s})\right\| \mathrm{d} s \leq\left\|\mathrm{u}_{0}\right\|+\Delta\left(\tilde{\mathrm{t}}-\mathrm{T}_{0}\right) .
$$

Then, $\left(\mathbf{u}_{\mathfrak{n}}(\tilde{\mathfrak{t}})\right)_{\mathfrak{n}}$ is relatively compact in $\mathbf{R}^{\mathfrak{n}}$, in addition, it is equi-continuous according to (32). By Arzelà-Ascoli theorem $\left(u_{n}\right)_{n}$ is relatively compact in $\mathcal{C}_{\mathbf{R}^{n}}\left(\left[T_{0}, \tau\right]\right)$, so, we can extract a subsequence of $\left(u_{n}\right)_{n}$ (that we do not relabel) which converges uniformly to some mapping $u$ on $\left[T_{0}, \tau\right]$. By the inequality $(32),\left(\dot{u}_{n}\right)_{n}$ converges in $\mathrm{L}_{\mathbf{R}^{n}}^{1}\left(\left[\mathrm{~T}_{0}, \tau\right]\right)$ to mapping $\dot{\mathrm{u}}(\cdot) \in \mathrm{L}_{\mathbf{R}^{n}}^{1}\left(\left[\mathrm{~T}_{0}, \tau\right]\right)$ with $\|\dot{\mathfrak{u}}(\tilde{\mathrm{t}})\| \leq \Delta$ a.e. $\tilde{\mathrm{t}} \in\left[\mathrm{T}_{0}, \tau\right]$. For the rest of the demonstration we can follow the proof of the part 1 to get

$$
\dot{\mathrm{u}}(\tilde{\mathrm{t}}) \in-\mathrm{N}_{\mathrm{C}(\tilde{\mathrm{t}}, \mathfrak{u}(\tilde{\mathrm{t}}))}(\mathfrak{u}(\tilde{\mathrm{t}}))+\mathrm{F}(\tilde{\mathrm{t}}, \mathfrak{u}(\tilde{\mathrm{t}}))+\mathrm{f}(\tilde{\mathrm{t}}, \mathfrak{u}(\tilde{\mathrm{t}})) \text {, a.e. } \tilde{\mathrm{t}} \in\left[\mathrm{T}_{0}, \tau\right] .
$$

Then, $\mathbf{S}_{\tau}\left(\mathbf{u}_{0}\right)$ is compact.

3) Now we show the upper semi-continuity of the set-valued mapping $\mathcal{R}_{\mathfrak{u}_{0}}(\cdot)$ on $\mathcal{I}$. Consider the graph of $\mathcal{R}_{\mathfrak{u}_{0}}(\cdot)$ defined by

$$
\operatorname{Gph}\left(\mathcal{R}_{\mathfrak{u}_{0}}\right)=\left\{(\tau, x) \in \mathcal{I} \times \mathbf{R}^{\mathfrak{n}}: x \in \mathcal{R}_{\mathfrak{u}_{0}}(\tau)\right\} .
$$

Let $\left(\tau_{n}, x_{n}\right) \in \operatorname{Gph}\left(\mathcal{R}_{u_{0}}\right)$ converges to $(\tau, x)$, then, for all $n \geq 0$ there exists a Lipschitz mapping $\left(u_{n}(\cdot)\right) \in S_{\tau}\left(u_{0}\right)$ such that $u_{n}\left(\tau_{n}\right)=x_{n} \in \mathcal{R}_{u_{0}}\left(\tau_{n}\right)$, by the compactness of $S_{\tau}\left(u_{0}\right)$ we can extract a subsequence of $\left(u_{n}(\cdot)\right)_{n}$ (that we do not relabel) which converges uniformly to the Lipschitz mapping $u(\cdot) \in S_{\tau}\left(u_{0}\right)$, and we have

$$
x=\lim _{n \rightarrow \infty} x_{n}=\lim _{n \rightarrow \infty} u_{n}\left(\tau_{n}\right)=u(\tau),
$$

so $x \in \mathcal{R}_{\mathfrak{u}_{0}}(\tau)$. We deduce that $\operatorname{Gph}\left(\mathcal{R}_{\mathfrak{u}_{0}}\right)$ is closed, then $\mathcal{R}_{\mathfrak{u}_{0}}(\cdot)$ is upper semi-continuous.

\section{Almost convex case}

In this section we study the existence of solution and a property of the attainable set to the perturbed sweeping process $(\mathcal{A S P})$, when we weaken the condition of convexity and upper semi-continuity. Then we present an exis-

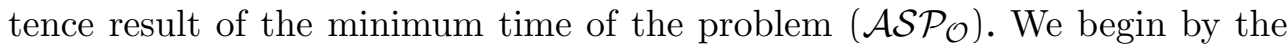
following preliminary lemma.

Lemma 2 Let $\mathrm{G}: \mathbf{R}^{\mathrm{n}} \rightarrow \mathbf{R}^{\mathrm{n}}$ be a measurable set valued mapping with nonempty compact and almost convex values. Then, there exist two integrable functions $\xi_{1}(\cdot)$ and $\xi_{2}(\cdot)$ defined on $\mathcal{I}$, satisfying $0 \leq \xi_{1}(t) \leq 1 \leq \xi_{2}(t)$ and for $\mathrm{t} \in \mathcal{I}$

$$
\xi_{1}(\mathrm{t}) \mathrm{m}(\mathrm{u}(\mathrm{t})) \in \mathrm{G}(\mathrm{u}(\mathrm{t})) \text { and } \xi_{2}(\mathrm{t}) \mathrm{m}(\mathrm{u}(\mathrm{t})) \in \mathrm{G}(\mathrm{u}(\mathrm{t})) \text {. }
$$


Proof. By the almost convexity of the values of $\mathrm{G}$ there exist two nonempty set-valued mappings $\Omega_{1}(\cdot)$ and $\Omega_{2}(\cdot)$ such that

$$
\Omega_{1}(t)=\left\{\xi_{1} \in[0,1]: \xi_{1} m(u(t)) \in G(u(t))\right\},
$$

and

$$
\Omega_{2}(t)=\left\{\xi _ { 2 } \in \left[1,+\infty\left[: \xi_{2} m(u(t)) \in G(u(t))\right\} .\right.\right.
$$

Let show that $\Omega_{1}(\cdot)$ is measurable. Consider its graph

$$
\operatorname{Gph}\left(\Omega_{1}\right)=\left\{\left(t, \xi_{1}\right) \in \mathcal{I} \times[0,1]: \xi_{1} m(u(t)) \in G(u(t))\right\},
$$

then,

$$
\begin{gathered}
\operatorname{Gph}\left(\Omega_{1}\right)=\left\{\left(t, \xi_{1}\right) \in \mathcal{I} \times[0,1]: d_{G(u(t))}\left(\xi_{1} m(u(t))\right)=0\right\} \\
=\sigma^{-1}(\{0\}) \cap(\mathcal{I} \times[0,1])
\end{gathered}
$$

where $\sigma:\left(t, \xi_{1}\right) \mapsto d_{G(u(t))}\left(\xi_{1} m(u(t))\right)$ is measurable. Then $\operatorname{Gph}\left(\Omega_{1}\right)$ is measurable. It follows that $\Omega_{1}$ is measurable on $\mathcal{I}$, then there exists a measurable selection $\xi_{1}(\cdot)$ defined on $\mathcal{I}$. The proof that $\Omega_{2}(\cdot)$ is measurable is similar, since $G(u(t))$ is bounded, and the same reasoning as in the previous point can be applied . Then, there exists measurable selection $\xi_{2}(\cdot)$ defined on $\mathcal{I}$.

Consider the following assumptions:

Assumption 1: Let C : $\mathbf{R}^{\mathrm{n}} \rightarrow \mathbf{R}^{\mathrm{n}}$ be a set-valued mapping with nonempty closed values satisfying:

$\left(\mathcal{H}_{1}^{C}\right)$ for all $x \in \mathbf{R}^{n}$, the sets $\mathrm{C}(x)$ are equi-uniformly subsmooth;

$\left(\mathcal{H}_{2}^{\mathrm{C}}\right)$ there is a constant $\mathrm{L}_{2} \in\left[0,1\left[\right.\right.$ and for any $x, u, v \in \mathbf{R}^{\mathfrak{n}}$ on has

$$
\left|d_{C(u)}(x)-d_{C(v)}(x)\right| \leq L_{2}\|u-v\| \text {. }
$$

Assumption 2: Let $\mathrm{F}: \mathbf{R}^{\mathfrak{n}} \rightarrow \mathbf{R}^{\mathfrak{n}}$ be a measurable set valued mapping with nonempty compact and almost convex values such that:

1. $\left(\mathcal{H}_{1}^{\mathrm{F}}\right)$ the set-valued mapping $\operatorname{co}(\mathrm{F}(\cdot))$ is upper semi-continuous on $\mathbf{R}^{\mathrm{n}}$;

2. $\left(\mathcal{H}_{2}^{\mathrm{F}}\right)$ for some real $\alpha>0, \mathrm{~d}_{\mathrm{co}(\mathrm{F}(\mathrm{x}))}(0) \leq \alpha(1+\|x\|)$ for all $x \in \mathbf{R}^{\mathrm{n}}$. 
Assumption 3: Let $\mathrm{f}: \mathbf{R}^{\mathrm{n}} \rightarrow \mathbf{R}^{\mathrm{n}}$ be a continuous mapping such that, for some real $\beta \geq 0$,

$$
\|f(x)\| \leq \beta(1+\|x\|), \forall x \in \mathbf{R}^{n} .
$$

Let

$$
\begin{aligned}
& X=\left\{F: \mathbf{R}^{\mathfrak{n}} \neg \mathbf{R}^{\mathfrak{n}}: \text { F satisfies Assumption } 1\right\}, \\
& Y=\left\{f \in \mathcal{C}_{\mathbf{R}^{\mathfrak{n}}}\left(\mathbf{R}^{\mathfrak{n}}\right): \text { f satisfies Assumption } 2\right\} .
\end{aligned}
$$

Since the class of almost convex sets is not stable under translation, we will define a larger class

$$
\mathrm{Z}=\{\mathrm{F} \in \mathrm{X}, \exists \mathrm{f} \in \mathrm{Y}: \mathrm{F}+\mathrm{f} \text { has almost convex values }\}
$$

which contains the set-valued mappings with almost convex values and their translated.

Theorem 2 Assume that the Assumption 1 holds and let $\mathrm{F} \in \mathrm{Z}$. Then, for every $\mathrm{u}_{0} \in \mathrm{C}\left(\mathrm{u}_{0}\right)$,

1. the problem $(\mathcal{A S P})$ admits a solution;

2. for all $\tau \in \mathcal{I}$, the attainable set of the problem $(\mathcal{A S P})$ at $\tau, \mathcal{R}_{\mathrm{u}_{0}}(\tau)$ coincides with $\mathcal{R}_{\mathfrak{u}_{0}}^{\text {co }}(\tau)$, the attainable set at $\tau$ of the convexified problem.

Proof. 1) (a) Let $[\alpha, \beta] \subset \mathcal{I}$ be an interval, and assume that, there exist two integrable functions $\xi_{1}(\cdot)$ and $\xi_{2}(\cdot)$ such that $0 \leq \xi_{1}(t) \leq 1 \leq \xi_{2}(t)$ for all $t \in[\alpha, \beta]$. In addition, assume that $\xi_{1}(\cdot)>0$ a.e., using the same technique as in the proof in [5] and [12], there exist two measurable subsets of $[\alpha, \beta]$, having characteristic functions $\chi_{1}$ and $\chi_{2}$ such that $\chi_{1}+\chi_{2}=\chi_{[\alpha, \beta]}$ and an absolutely continuous function $y=y(t)$ on $[\alpha, \beta]$, such that

$$
\dot{y}(t)=\frac{1}{\xi_{1}(t)} x_{1}(t)+\frac{1}{\xi_{2}(t)} x_{2}(t) \text { and } y(\beta)-y(\alpha)=\beta-\alpha .
$$

(b) By Theorem 1 there exists a Lipschitz solution $x: \mathcal{I} \rightarrow \mathbf{R}^{\mathrm{n}}$ of the convexified problem

$$
\left(\mathcal{A S P}_{\text {co }}\right) \quad\left\{\begin{array}{l}
\dot{\mathrm{u}}(\mathrm{t}) \in-\mathrm{N}_{\mathrm{C}(\mathrm{u}(\mathrm{t}))}(\mathrm{u}(\mathrm{t}))+\operatorname{co}(\mathrm{F}(\mathrm{u}(\mathrm{t})))+\mathrm{f}(\mathrm{u}(\mathrm{t})), \quad \text { a.e. } \mathrm{t} \in \mathcal{I} ; \\
\mathrm{u}(\mathrm{t}) \in \mathrm{C}(\mathrm{u}(\mathrm{t})), \quad \forall \mathrm{t} \in \mathcal{I} ; \quad \mathrm{u}\left(\mathrm{T}_{0}\right)=\mathrm{u}_{0} \in \mathrm{C}\left(\mathrm{u}_{0}\right) .
\end{array}\right.
$$

Let set $\mathrm{m}_{\mathrm{T}}(x(\tau))=\operatorname{Proj}_{\mathrm{co}(\mathrm{F}(x(\tau)))+\mathrm{f}(x(\tau)}(0)$ and consider the closed set

$$
\mathcal{A}=\left\{\tau \in \mathcal{I}: \mathrm{m}_{\mathrm{T}}(\chi(\tau))=0\right\} .
$$


Case 1: $\mathcal{A}$ is empty. In this case $\xi_{1}(\tau)>0$, so, we can apply the part (a) to the interval $\mathcal{I}$. Set $y(\tau)=T_{0}+\int_{T_{0}}^{\tau} \dot{y}(s) d s$ is increasing and we have $y\left(T_{0}\right)=$ $\mathrm{T}_{0}$ and $\mathrm{y}(\mathrm{T})=\mathrm{T}$, so, $y$ defined from $\mathcal{I}$ into itself. Let $\vartheta: \mathcal{I} \rightarrow \mathcal{I}$ be its inverse, then $\vartheta\left(T_{0}\right)=T_{0}, \vartheta(T)=T, 1=\dot{y}(\vartheta(\tau)) \dot{\vartheta}(\tau)$ and

$$
\dot{\vartheta}(\tau)=\xi_{1}(\vartheta(\tau)) \chi_{1}(\tau)+\xi_{2}(\vartheta(\tau)) \chi_{2}(\tau) .
$$

Define the map $\tilde{x}: \mathcal{I} \rightarrow \mathbf{R}^{n}$, as $\tilde{x}(\tau)=\chi(\vartheta(\tau))$ for all $\tau \in \mathcal{I}$, then we have

$$
\frac{\mathrm{d}}{\mathrm{d} \tau} \tilde{x}(\tau)=\dot{\vartheta}(\tau) \frac{\mathrm{d}}{\mathrm{d} \tau} \chi(\vartheta(\tau)) \in \dot{\vartheta}(\tau)\left(-\mathrm{N}_{\mathrm{C}(x(\vartheta(\tau)))}(x(\vartheta(\tau)))+\mathrm{m}_{\mathrm{T}}(x(\vartheta(\tau))),\right.
$$

using the property of the normal cone and the definition of the set Z. we get, far all $\tau \in \mathcal{I}$

$$
\begin{aligned}
\frac{\mathrm{d}}{\mathrm{d} \tau} \tilde{x}(\tau) & \in-\mathrm{N}_{\mathrm{C}(x(\vartheta(\tau)))}(x(\vartheta(\tau)))+\mathrm{F}(x(\vartheta(\tau)))+\mathrm{f}(x(\vartheta(\tau))) \\
& =-\mathrm{N}_{\mathrm{C}(\tilde{x}(\tau))}(\tilde{x}(\tau))+\mathrm{F}(\tilde{x}(\tau))+\mathrm{f}(\tilde{x}(\tau)) .
\end{aligned}
$$

Case 2: $\mathcal{A}$ is non-empty. Let $\mathrm{c}=\sup \{\tau, \tau \in \mathcal{A}\}$, so that $\mathrm{c} \in \mathcal{A}$ because $\mathcal{A}$ is closed relative to $\mathcal{I}$. The complement of $\mathcal{A}$ is open relative to $\mathcal{I}$, it consists of at most countably many overlapping open intervals $] \alpha_{i}, \beta_{i}[$, with the possible exception of one of the form $\left[c, \beta_{i_{i}}\right.$. For each $i$, apply part (a) to the interval ]$\alpha_{i}, \beta_{i}[$, to infer the existence of two measurable subsets of $] \alpha_{i}, \beta_{i}[$ with characteristic functions $\chi_{1}^{i}(\cdot)$ and $\chi_{2}^{i}(\cdot)$ such that $\chi_{1}^{i}(\cdot)+\chi_{2}^{i}(\cdot)=\chi_{] \alpha_{i}, \beta_{i}[}(\cdot)$. Setting, $\dot{y}(\tau)=\frac{1}{\xi_{1}(\tau)} \chi_{1}^{i}(\tau)+\frac{1}{\xi_{2}(\tau)} x_{2}^{i}(\tau)$, we obtain $\int_{\alpha_{i}}^{\beta_{i}} \dot{y}(\tau) d \tau=\beta_{i}-\alpha_{i}$.

On $\left[T_{0}, c\right]$, set

$$
\dot{y}(\tau)=\frac{1}{\xi_{2}(\tau)} \chi_{\mathcal{A}}(\tau)+\sum_{i}\left(\frac{1}{\xi_{1}(\tau)} \chi_{1}^{i}(\tau)+\frac{1}{\xi_{2}(\tau)} \chi_{2}^{i}(\tau)\right),
$$

where the sum is over all intervals contained in $\left[T_{0}, c\right]$, in addition to that $\xi_{2}(\tau) \geq 1$ and $\int_{T_{0}}^{c} \dot{y}(\tau) d \tau=\kappa \leq c-T_{0}$. Setting $y(\tau)=T_{0}+\int_{T_{0}}^{\tau} \dot{y}(\tau) d \tau$, we obtain that $y(\cdot)$ is an invertible map from $\left[T_{0}, c\right]$ to $\left[T_{0}, k\right]$. Define $\vartheta=\vartheta(\tau)$ from $\left[T_{0}, k\right]$ to $\left[T_{0}, c\right]$ to be the inverse of $y(\cdot)$, then extend $\vartheta(\cdot)$ as an absolutely continuous map $\tilde{\vartheta}(\cdot)$ on $\left[T_{0}, c\right]$. Setting $\dot{\tilde{\vartheta}}(\tau)=0$ for all $\left.\left.\tau \in\right] \kappa, c\right]$. We prove the mapping $\tilde{x}(\tau)=\chi(\tilde{\vartheta}(\tau))$ is a solution of the problem $(\mathcal{A S P})$ on $\left[T_{0}, c\right]$ satisfying $\tilde{x}(c)=x(c)$.

For $\tau \in\left[T_{0}, \kappa\right]$, we get $\tilde{\vartheta}(\tau)=\vartheta(\tau)$ it is invertible and

$$
\dot{\vartheta}(\tau)=\xi_{2}(\vartheta(\tau)) \chi_{A}(\vartheta(\tau))+\sum_{i}\left(\xi_{1}(\vartheta(\tau)) \chi_{1}^{i}(\vartheta(\tau))+\xi_{2}(\vartheta(\tau)) \chi_{2}^{i}(\vartheta(\tau))\right) .
$$


As $\frac{d}{d \tau} \tilde{\chi}(\tau)=\dot{\vartheta}(\tau) \frac{d}{d \tau} \chi(\vartheta(\tau))$, we have

$$
\frac{\mathrm{d}}{\mathrm{d} \tau} \tilde{x}(\tau)=\dot{\vartheta}(\tau)\left(-\mathrm{N}_{\mathrm{C}(\mathrm{x}(\vartheta(\tau)))}(x(\vartheta(\tau)))+\mathrm{m}_{\mathrm{T}}(x(\tau))\right) .
$$

Using (33) and the properties of the normal cone we get

$$
\begin{aligned}
\frac{\mathrm{d}}{\mathrm{d} \tau} \tilde{x}(\tau) & \in-\mathrm{N}_{\mathrm{C}(x(\vartheta(\tau)))}(x(\vartheta(\tau)))+\mathrm{F}(x(\vartheta(\tau)))+\mathrm{f}(x(\vartheta(\tau))) \\
& \in-\mathrm{N}_{C(\tilde{x}(\tau))}(\tilde{x}(\tau))+\mathrm{F}(\tilde{x}(\tau))+\mathrm{f}(\tilde{x}(\tau))
\end{aligned}
$$

For $\tau \in] \kappa, c]$, we get $\vartheta(\kappa)=\mathrm{c}$ and $\dot{\tilde{\vartheta}}(\tau)=0$, then we have $\tilde{\vartheta}(\tau)=\tilde{\vartheta}(\kappa)=\vartheta(\kappa)$, so $\tilde{x}(\tau)=\chi(\tilde{\vartheta}(\tau))=\chi(\tilde{\vartheta}(\kappa))=\tilde{x}(\kappa)$, then $\tilde{x}$ is constant on $] \kappa, c]$ and we have $\frac{\mathrm{d}}{\mathrm{d} \tau} \tilde{\mathrm{x}}(\tau)=0 \in \operatorname{co}\left(\mathrm{F}(\tilde{\mathrm{x}}(\tau))+\mathrm{f}(\tilde{\mathrm{x}}(\tau))\right.$, in addition $0 \in \mathrm{N}_{\mathrm{C}(\tilde{\mathrm{x}}(\tau))}(\tilde{x}(\tau))$, we conclude that for all $\tau \in] \kappa, c]$

$$
\frac{\mathrm{d}}{\mathrm{d} \tau} \tilde{x}(\tau)=0 \in-\mathrm{N}_{\mathrm{C}(\tilde{\mathrm{x}}(\tau))}(\tilde{x}(\tau))+\mathrm{F}(\tilde{\mathrm{x}}(\tau))+\mathrm{f}(\tilde{\mathrm{x}}(\tau))
$$

On $] c, T], \mathcal{A}$ is empty and $\xi_{1}(\tau)>0$, then we can repeat the arguments of the part (a). We conclude, That $\tilde{x}$ is a solution of the problem $(\mathcal{A S P})$.

2) For all $\tau \in \mathcal{I}, \mathcal{R}_{\mathfrak{u}_{0}}(\tau) \subset \mathcal{R}_{\mathfrak{u}_{0}}^{c o}(\tau)$. It is enough to prove the converse inclusion.

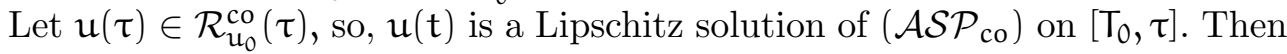
the proof of Theorem 2 can be repeated on $\left[T_{0}, v\right]$ and we find a solution $\tilde{\mathfrak{u}}(\cdot):\left[\mathrm{T}_{0}, \mathcal{v}\right] \rightarrow \mathbf{R}^{\mathrm{n}}$ of the problem $(\mathcal{A S P})$ such that $\tilde{\mathfrak{u}}(\tau)=\mathfrak{u}(\tau) \in \mathcal{R}_{\mathfrak{u}_{0}}(\mathrm{t})$. Then $\mathcal{R}_{\mathfrak{u}_{0}}^{\text {co }}(\mathrm{t}) \subset \mathcal{R}_{\mathfrak{u}_{0}}(\mathrm{t})$. Hence we get the needed coincidence.

The following corollary to Theorem 2, to be compared with Theorem 1 of Filippov [20], shows that, in the case of autonomous control systems, for the existence of a time optimal solution, Filippov's assumption that the set $h(x, U(x))$ is convex can be replaced by the weaker assumption that the same set is almost convex.

Corollary 1 Assume that Assumption 1 holds. Let $\mathrm{U}: \mathbb{R}^{n} \rightrightarrows \mathbb{R}^{n}$ be a setvalued mapping with compact valued that is upper semi-continuous on $\mathbb{R}^{n}$ and $\mathrm{h}: \mathbb{R}^{\mathrm{n}} \times \mathbb{R}^{\mathrm{n}} \rightarrow \mathbb{R}^{\mathrm{n}}$ be a continuous mapping satisfying the following assumption

$\left(\mathcal{H}^{\mathrm{h}}\right)$ there is a nonnegative constant $\alpha$, such that $\|\mathrm{h}(\mathrm{x}, \mathrm{y})\| \leq \alpha(1+\|\mathrm{x}\|)$, for all $(x, y) \in \mathbb{R}^{n} \times \mathbb{R}^{n}$ 
We associate with these data the set-valued mapping $\mathrm{F}: \mathbf{R}^{\mathrm{n}} \rightarrow \mathbf{R}^{\mathrm{n}}$ defined by

$$
\mathrm{F}(\mathrm{x})=\{\mathrm{h}(\mathrm{x}, z)\}_{z \in \mathrm{U}(\mathrm{x})}, \text { for all } \mathrm{x} \in \mathbb{R}^{\mathrm{n}} .
$$

Assume that $\mathrm{F} \in \mathrm{Z}$, where

$$
\mathrm{Z}=\{\mathrm{F} \in \mathrm{X}, \exists \mathrm{f} \in \mathrm{Y}: \mathrm{F}+\mathrm{f} \text { has almost convex values }\} .
$$

Let $\mathrm{u}_{0}$ and $\zeta$ be given in $\mathbf{R}^{\mathrm{n}}$ such that $\mathrm{u}_{0} \in \mathrm{C}\left(\mathrm{u}_{0}\right)$ and for some $\overline{\mathrm{t}} \in\left[\mathrm{T}_{0}, \mathrm{~T}\right]$, $\zeta \in \mathcal{R}_{\mathfrak{u}_{0}}(\overline{\mathfrak{t}})$. Then, the problem of reaching $\zeta$ from $\mathbf{u}_{0}$ in a minimum time admits a solution.

Proof. Consider

$$
\mathcal{M}=\left\{\mathrm{t} \in\left[\mathrm{T}_{0}, \overline{\mathrm{t}}\right]: \zeta \in \mathcal{R}_{\mathrm{u}_{0}}(\mathrm{t})\right\} .
$$

By hypothesis $\mathcal{M} \neq \emptyset$. We put $\tau=\inf \mathcal{M}$, then, there exists a decreasing sequence $\left(\tau_{\mathfrak{n}}\right)$ in $\left[T_{0}, \bar{t}\right]$ converges to $\tau$, and a mapping $u_{n}(\cdot)$ solution of

$$
\left\{\begin{array}{l}
\dot{u}(t) \in-N_{C}(u(t))(u(t))+F(u(t))+f(u(t)) \text { a.e. } t \in\left[T_{0}, \tau_{n}\right] ; \\
u(t) \in C(u(t)), \forall t \in\left[T_{0}, \tau_{n}\right] ; u\left(T_{0}\right)=u_{0} \in C\left(u_{0}\right) .
\end{array}\right.
$$

such that for all $n \geq 1, u_{n}\left(\tau_{n}\right)=\zeta$. Also, for all $n \geq 1, u_{n}(\cdot)$ is solution of

$$
\left\{\begin{array}{l}
\dot{u}(t) \in-N_{C}(u(t))(u(t))+c o(F)(u(t))+f(u(t)) \quad \text { a.e. } t \in\left[T_{0}, \tau_{n}\right] \\
u(t) \in C(u(t)), \forall t \in\left[T_{0}, \tau_{n}\right] ; u\left(T_{0}\right)=u_{0} \in C\left(u_{0}\right) .
\end{array}\right.
$$

Let $w_{n}(t)=u_{n}(t)$ for $t \in[0, \tau]$ and $n \geq 1, w_{n}(\cdot) \in S_{\tau}\left(u_{0}\right)$, by the proof of theorem 2 this set is compact, then by extracting a subsequence if necessary we may conclude that $\left(w_{n}(\cdot)\right.$ converges uniformly to $w(\cdot) \in S_{\tau}\left(u_{0}\right)$. On the other hand, we have $\zeta=\mathfrak{u}_{n}\left(\tau_{n}\right) \in \mathcal{R}_{\mathfrak{u}_{0}}^{\text {co }}\left(\tau_{n}\right)$, by Theorem 2 again, the multifunction $\mathcal{R}_{\mathfrak{u}_{0}}^{\text {co }}(\cdot)$ is upper semi-continuous with nonempty compact values, so we get $\limsup \mathcal{R}_{\mathfrak{u}_{0}}^{\text {co }}\left(\tau_{\mathfrak{n}}\right)=\mathcal{R}_{\mathfrak{u}_{0}}^{\text {co }}(\tau)$. Then, $\zeta \in \mathcal{R}_{\mathfrak{u}_{0}}^{\text {co }}(\tau)=\mathcal{R}_{\mathfrak{u}_{0}}(\tau)$. Consequently, $w$ is the solution of the problem $\left(\mathcal{A S P}_{\mathcal{O}}\right)$ that reaches $\zeta$ in the minimum time, and $\tau$ is the value of the minimum time.

\section{Acknowledgements}

Research supported by the General direction of scientific research and technological development (DGRSDT) under project PRFU No. C00L03UN 180120180001. 


\section{References}

[1] S. Adly, F. Nacry and L. Thibault, Discontinuous sweeping process with prox-regular sets, ESAIM Control Optim. Calc. Var., 23 (2017), 12931329.

[2] K. Addi, B. Brogliato and D. Goeleven, Aqualitative mathematical analysis of a class of linear variational inequalities via semi-complementarity problems, Appl. Electron. Math. Program., 126 (2011), 31-67.

[3] D. Affane, M. Aissous and M. F. Yarou, Existence results for sweeping process with almost convex perturbation, Bull. Math. Soc. Sci. Math. Roumanie, 2 (2018), 119-134.

[4] D. Affane, M. Aissous and M. F. Yarou, Almost mixed semi-continuous perturbation of Moreau's sweeping process, Evol. Equ. Control Theory, 1 (2020), 27-38.

[5] D. Affane and D. Azzam-Laouir, Almost convex valued perturbation to time optimal control sweeping processes, ESAIM Control Optim. Calc. Var, 23 (2017), 1-12.

[6] D. Aussel, A Danilis and L. Thibault, Subsmooth sets: functional characterizations and related concepts, Trans. Amer. Math. Soc. 357 (2004), $1275-1301$.

[7] H. Benabdellah, Existence of solutions to the nonconvex sweeping process, J. Differential Equations, 164 (2000), 286-295.

[8] M. Bounkhel and M. F. Yarou, Existence results for first and second order nonconvex sweeping processs with delay, Port. Math., 61 (2004), 207-230.

[9] C. Castaing, A. G. Ibrahim and M. F. Yarou , Some contributions to nonconvex sweeping process, J. Nonlinear Convex Anal., 10 (2009), 120.

[10] N. Chemetov and M. D. P. Monteiro Marques, Non-convex Quasivariational Differential Inclusions, Set-Valued Var. Anal., 5 (2007), 209221.

[11] K. Chraibi, Resolution du problème de rafle et application a un problème de frottement, Topol. Methods Nonlinear Anal., 18 (2001), 89-102. 
[12] A. Cellina and A. Ornelas, Existence of solution to differential inclusion and the time optimal control problems in the autonomous case, SIAM J. Control Optim., 42 (2003), 260-265.

[13] F. H. Clarke, Y. S. Ledyaev, R. J. Stern and P. R. Wolenski, Nonsmooth Analysis and Control Theory, Springer, New York, 1998.

[14] G. Colombo and V. V. Goncharov, The sweeping processes without convexity, Set-Valued Anal., 18 (1999), 357-374.

[15] G. Colombo, R. Henrion, N. D Hoang and B. Sh. Mordukhovich, Discrete approximations of a controlled sweeping process, Set-Valued Var. Anal., 23 (2015), 69-86.

[16] G. Colombo, R. Henrion, N. D Hoang and B. Sh. Mordukhovich, Optimal control of the sweeping process over polyhedral controlled sets, J. Differ. Equ., 260 (2016), 3397-3447.

[17] G. Colombo and L. Thibault, Prox-regular sets and applications, in Handbook of Nonconvex Analysis and Applications, International PressSomerville, MA, (2010), 99-182.

[18] S. Di Marino, F. Santambrogio and B. Maury, Measure sweeping processes, J. Conv. An., 23 (2015), 567-601.

[19] D. Goeleven, Complementarity and Variational Inequalities in Electronics, Mathematical Analysis and its Applications, Academic Press, London, 2017.

[20] A. F. Filippov, On certain questions in the theory of optimal control, Vestnik. Univ., Ser. Mat. Mech., 2 (1959), 25-32; Translated in SIAM J. Control, 1 (1962), 76-84.

[21] T. Haddad, J. Noel and L. Thibault, Perturbation sweeping process with a subsmooth set depending on the state,Linear Nonlinear Anal., 2 (2016), $155-174$.

[22] M. Kunze and M. D. P. Monteiro Marques, An introduction to Moreau's sweeping process, in B. Brogliato, (ed.) Impacts in Mechanical Systems. Analysis and Modelling, Springer, Berlin, 2000 1-60.

[23] B. Sh. Mordukhovich and Y. Shao, Nonsmooth sequential analysis in asplund space, Trans. Amer. Math. Soc., 4 (1996), 1235-1279. 
[24] J. J. Moreau, Rafle par un convexe variable I, Sem. Anal. Convexe Montpellier, exposé No. 15 (1971).

[25] J. J. Moreau, Rafle par un convexe variable II, Sem. Anal. Convexe Montpellier, exposé No. 3 (1972).

[26] J. J. Moreau, Evolution problem associated with amoving convex set in a Hilbert Space, J. Differ. Equ., 26 (1977), 347-374.

[27] J. J. Moreau, Unilateral contact and dry friction in finite freedom dynamics, Nonsmooth Mechanics and Applications 302 in CISM, Courses and Lectures, Springer Verlag, 1988.

[28] V. Recupero, A continuity method for sweeping processes, J. Differ. Equ., 251 (2011), 2125-2142 .

[29] V. Recupero, BV continuous sweeping processes, J. Differ. Equ., 259 (2015), 4253-4272.

[30] V. Recupero, Sweeping processes and rate independence, J. Convex Anal., 23 (2016), 921-946.

[31] V. Recupero, F. Santambrogio, Sweeping processes with prescribed behavior on jumps, Ann. Mat. Pura Appl., 197 (2018), 1311-1332.

Received: December 25, 2020 